\title{
A new parameterization of the reionisation history
}

\author{
M. Douspis ${ }^{1}$, N. Aghanim ${ }^{1}$, S. Ilicí ${ }^{1,2}$, and M. Langer ${ }^{1}$ \\ 1 Institut d'Astrophysique Spatiale, Université Paris-Sud, CNRS, UMR8617, 91405 Orsay, France \\ e-mail: marian.douspis@ias.u-psud.fr \\ 2 IRAP, Université de Toulouse, UPS-OMP, CNRS, 14 avenue Edouard Belin, 31400 Toulouse, France
}

Received 17 May 2015 / Accepted 28 June 2015

ABSTRACT

\begin{abstract}
Motivated by the current constraints on the epoch of reionisation from recent cosmic microwave background observations, ionising background measurements of star-forming galaxies, and low redshifts line-of-sight probes, we propose a new data-motivated parameterisation of the history of the average ionisation fraction. This parameterisation describes a flexible redshift-asymmetric reionisation process in two regimes that is capable of fitting all the current constraints.
\end{abstract}

Key words. dark ages, reionization, first stars - large-scale structure of Universe - cosmic background radiation

\section{Introduction}

After recombination at $z \approx 1090$, the Universe was essentially neutral. Observations of the Gunn-Peterson (GP) effect (Gunn $\&$ Peterson 1965) in quasar spectra (e.g. Fan et al. 2006) indicate that intergalactic gas has become almost fully reionised by redshift $z \sim 6$. This transition, called cosmic reionisation, is one of the most important events in cosmic structure formation and in cosmology. It is also of particular importance since it may be related to many fundamental questions, such as the presence of annihilating or decaying dark matter particles, properties of the first galaxies, physics of (mini-)quasars, and the formation of very metal-poor stars. The reionisation process starts with the formation of the first generation of early star-forming galaxies and quasars that emit ultraviolet radiation that reionises the neutral regions around them. This is the so-called patchy reionisation stage (Aghanim et al. 1996). After a sufficient number of ionising sources have formed and ionised regions have overlapped, the ionised portion of the gas in the Universe rapidly increases until hydrogen becomes fully ionised. This period, during which the cosmic gas went from neutral to ionised, is known as the Epoch of Reionisation (EoR). The transition from neutral to ionised diffuse gas is still not observed directly but is constrained by the absorption spectra of very distant quasars and gamma ray bursts, which reveal neutral hydrogen in intergalactic clouds. These GP effect observations show that the diffuse gas in the Universe is mostly ionised up to a redshift of order 6 (Fan et al. 2006).

The reionisation process describes the balance between the recombination of free electrons with protons to form neutral hydrogen and the ionisation of hydrogen atoms by photons with energies $h v>13.6 \mathrm{eV}$. The first empirical, analytic, and numerical models of the reionisation process (e.g. Aghanim et al. 1996; Gruzinov \& Hu 1998; Madau et al. 1999; Gnedin 2000; Ciardi et al. 2003) highlighted the basic physics that give rise to the ionised intergalactic medium (IGM) at late times and provided predictions of the effects on the cosmic microwave background $(\mathrm{CMB})$. The reionisation history is conveniently expressed in terms of the filling factor of ionised hydrogen $Q_{\mathrm{HII}}$. One of the relevant physical and the most commonly used quantities for characterising reionisation is the Thomson scattering optical depth to the CMB $\tau=\int_{\eta_{\mathrm{CMB}}}^{\eta_{0}} a n_{\mathrm{e}} \sigma_{\mathrm{T}} \mathrm{d} \eta$, where $n_{\mathrm{e}}$ is the number density of free electrons at a conformal time $\eta, \sigma_{\mathrm{T}}$ is the Thomson scattering cross-section, $a$ the scale factor, and $\eta_{0}$ the conformal time today.

Reionisation leaves imprints on the $\mathrm{CMB}$ power spectra, both in polarisation and in intensity, and through the kinetic Sunyaev-Zeldovich (kSZ) effect, due to the re-scattering of photons off free electrons (see Aghanim et al. 2008, and references therein). The temperature power spectrum is damped at scales larger than the horizon. This effect is degenerate with the amplitude $A_{\mathrm{s}}$ and slope $n_{\mathrm{s}}$ of the initial power spectrum. The CMB temperature thus constrains the amplitude $A_{\mathrm{s}} e^{-2 \tau}$. The large-scale polarisation spectra present a reionisation "bump" that WMAP first measured in TE in 2003 (Spergel et al. 2003). In fact, $\mathrm{CMB}$ observations allow us to shed light only on the Thomson optical depth $\tau$. In addition to the CMB, other astrophysical probes constrain reionisation.

Direct measurements of the ionisation state of the Universe through $\operatorname{Ly} \alpha$ emission or quasars, between redshift 5 and redshift 10, have improved in quality and statistics (e.g. Fan et al. 2006; Faisst et al. 2014). Ionising background estimates from star-forming galaxies improved as well in parallel to the depth of observations (e.g. Ishigaki et al. 2015; Robertson et al. 2015; Bouwens et al. 2015, hereafter ISHI15, ROB15, BOU15, respectively). New probes are becoming available and increasingly useful, such as the $21 \mathrm{~cm}$ brightness fluctuations (e.g. Bowman \& Rogers 2010) or gamma-ray bursts (e.g. Chornock et al. 2014). While it was hard to reconcile the ionising power of galaxies with the high value of $\tau$ derived from WMAP (Spergel et al. 2003), the latest results from Planck (Planck Collaboration XIII 2015, hereafter PCP15) give an almost coherent picture of the EoR where star-forming galaxies play a dominant role in ionising the Universe.

Large numbers of detailed models of the reionisation history have been constructed to describe the transition from a neutral to 
an ionised state and to reproduce the EoR observables (Pritchard et al. 2010; Pandolfi et al. 2011; Mitra et al. 2011, 2012, 2015). These physically motivated models need to make assumptions on various complex processes (e.g. radiative transfer, star formation and evolution, chemical enrichement) captured in effective parameters, such as escape fraction, clumping factor, and star formation efficiency. As succesful as they are, those models cannot be used easily to explore the global history of the reionisation transition rapidly and without any prior on the detailed physics.

In this Letter, therefore, we provide a new parameterisation of the mean ionised fraction of the Universe as a function of redshift. We exploit in particular the recent results based on the measurements of UV and IR flux of early galaxies in order to derive a simple data-motivated, fast, and economic expression of the ionised fraction. It agrees with the current observational constraints on EoR better than the standard parametisation used in CMB analyses.

\section{Observational status and current constraints on reionisation}

The reionisation process is intimately linked to the formation and evolution of the first emitting sources. At redshifts as high as 15 to 30, low-mass halos of matter detach from the general expansion, collapse, and virialise. If they reach high enough temperatures to excite the lowest electronic levels of hydrogen, they cool and eventually form Population III stars and dwarf atomiccooling galaxies. These emitting sources, thought to have initiated the reionisation process, have different various properties (masses, soft/hard spectra, UV escape fractions, etc.). The reionisation process is thus quite complex since it implies multiple sources of UV photons that combine their relative efficiencies at different epochs and scales.

The abundance of quasars declines rapidly beyond redshift $z \sim 6$. Quasars therefore cannot be significant contributors to reionisation at high redshifts (e.g. Willott et al. 2010; Fontanot et al. 2012), but they are key players at lower redshifts since they ensure the second reionisation of helium (McQuinn et al. 2009). Faint star-forming galaxies at redshifts $z \gtrsim 6$ have therefore been postulated as the most likely sources of reionisation, and their time-dependent abundance and spectral properties are crucial ingredients for understanding how intergalactic hydrogen became reionised (for reviews, see Fan et al. 2006; Robertson et al. 2010; Loeb \& Furlanetto 2013). The high-redshift galaxy UV luminosity function also provides a competitive observational constraint on EoR. Based on the 2012 Hubble Ultra Deep Field galaxies at $z \sim 7-12$, Ellis et al. (2013) show a continuous decline in the abundance of star-forming galaxies over $6<z<10$, whereas Dunlop et al. (2013), based on the constancy of the UV continuum slope measured in $z \simeq 7-9$ galaxies over a wide range in luminosity, show that stars at these redshifts are already enriched by earlier generations, supporting the idea of a reionisation process that extended beyond $z=9$. Finally, Robertson et al. (2013) combined the results from UDF12 with the measured Thomson optical depth and stellar mass density measurements (Stark et al. 2013) and provided constraints on the role of high-redshift starforming galaxies in the reionisation process. Under reasonable assumptions about the escape fraction of ionising photons and the IGM clumping factor, they find that the observed galaxy population $\left(M_{U V}<-17\right.$ at $\left.z \sim 8\right)$ cannot simultaneously reionise the universe by $z \sim 6$ and produce a large Thomson optical depth, unless the abundance of star-forming galaxies and/or the ionising photon escape fraction increase in the range $z \sim 15-25$ with respect to what is currently observed (see e.g. Kuhlen $\&$ Faucher-Giguère 2012). The star formation rate (SFR) history allows the duration of reionisation to be probed (Madau \& Dickinson 2014) because star-forming galaxies provide most of the ionising photons (Robertson et al. 2013). Recent works by ISHI15, ROB15, and BOU15 (see also references therein) have shown that star-forming galaxies at $z \sim 8-10$ were abundant enough to ionise the Universe and yield a Thomson optical depth of the order of 0.06 .

CMB data can also constrain reionisation history through secondary anisotropies in temperature and polarisation. Measuring the latter has proven to be a challenging task because the signal is strongly contaminated by both foregrounds and systematics on large angular scales (Planck Collaboration Int. XXX 2015). This has affected the accuracy of optical depth measurements (Spergel et al. 2003; Page et al. 2007) and limited its precision (Hinshaw et al. 2013, PCP15). Recent results from Planck combining temperature and polarisation provide $\tau=0.078 \pm 0.019$ in the $\Lambda$-CDM model (PCP15). The degeneracy between $A_{\mathrm{s}}$ and $\tau$ can be broken by adding information about lensing (Planck Collaboration XVI 2014). Planck also provides $\tau=0.66 \pm 0.013$ when considering temperature, polarisation, lensing, and baryonic acoustic oscillations (BAO) in $\Lambda$-CDM (Eq. (17e) of PCP15, hereafter referred to as the Planck reference). For an instantaneous reionisation, this corresponds to a redshift of $z_{\mathrm{re}}=8.8_{-1,2}^{1.1}$. Extending the standard $\Lambda$-CDM model probes reionisation history in a broader range of cosmologies. Two extreme cases for $\tau$ are referred to in the following as high- $\tau$ and low- $\tau$, corresponding to a MCMC run using both temperature and polarisation at high and low multipoles leaving the amplitude of lensing free and a run with temperature only at high multipoles and temperature and polarisation at low multipoles with curvature free, respectively ${ }^{1}$. The two corresponding optical depth values are: $\tau \sim 0.11 \pm 0.03$ and $\tau \sim 0.054 \pm 0.021$.

We show in Fig. 1 (upper panel) the current data and constraints from direct observations of the ionised hydrogen fraction $Q_{\mathrm{HII}}$ (symbols, from BOU15 and references therein), CMB (solid lines), and star-forming high-redshift galaxies (dashed lines); namely, we plot the measurements from line-of-sight observations as compiled in ROB 15. We further add the lower limit estimates of the neutral hydrogen fraction at $z \sim 7.7$ from two additional Ly- $\alpha$ emitters (Faisst et al. 2014) and the neutral fraction estimate from the optical afterglow of the Swift gamma-ray burst (GRB) 140515A at $z \sim 6.15$ (Chornock et al. 2014). The high- $\tau$ constraint from CMB (blue shaded area) is incompatible with other reionisation constraints. We furthermore note that within all the possible CMB models, those providing us with low optical depths agree best with the data points. However, changes in $\tau$ only shift the best-fit CMB curves to high or low redshifts without modifying their shapes. As a result, no satisfactory fit seems attainable with the standard reionisation history used in $\mathrm{CMB}$ analyses. Figure 1 (middle panel) displays the evolution of the SFR density $\rho_{\text {SFR }}$ compiled by ROB 15 . Overplotted are the best models from Robertson et al. (2015) (dashed line) and the $\rho_{\mathrm{SFR}}$ derived from the best-fit CMB models $^{2}$ with increasing values of $\tau$ from left (solid green curve) to right (solid light-blue curve). The dashed grey line shows

1 MCMC runs named base_omegak_plikHM_TT_lowTEB and base_Alens_plikHM_TTTEEE_lowEB are available on Planck Legacy Archive: http://pla.esac.esa.int/pla/

2 To convert from $\dot{N}_{\text {ion }}$ to $\rho_{\mathrm{SFR}}$, we assume $\log _{10}\left(\left\langle f_{\text {esc }} \xi_{\text {ion }}\right\rangle\right)=52.44$ as in ROB15. 

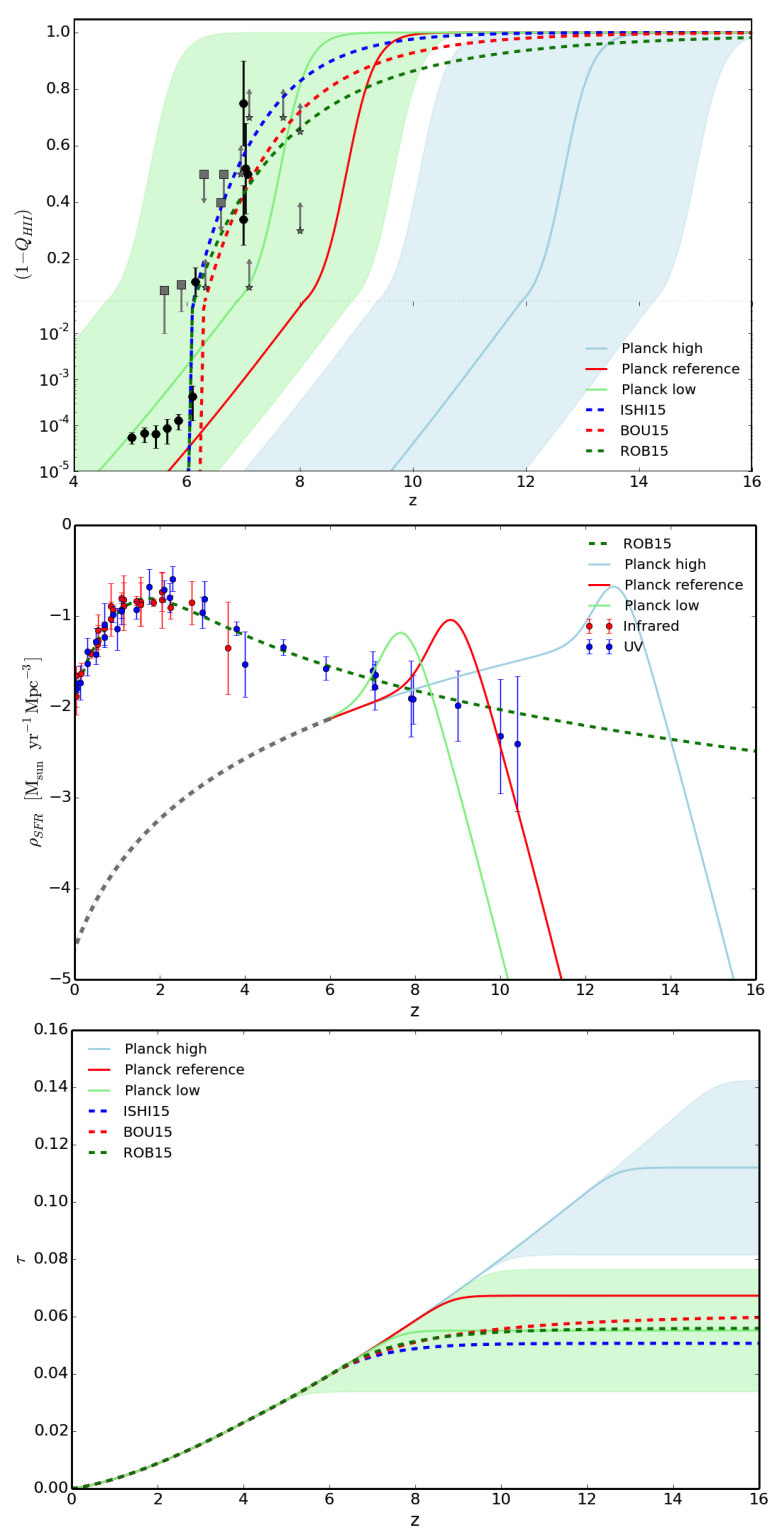

Fig. 1. Upper panel: reionisation histories compared to low-redshift probes. Shaded envelopes show the low/high- $\tau$ values derived from Planck with uncertainties. The red line indicates the best Planck value $\tau=0.66$. Dashed lines are derived from the best ionisation rate $\left(\dot{N}_{\text {ion }}\right)$ of ISHI15, ROB15, BOU15. Symbols are current constraints from direct line-of-sight measurements compiled by BOU15. Middle panel: corresponding $\rho_{\mathrm{SFR}}$ for Planck and the best fit of ROB15 with observational data compiled by ROB 15 . The grey dashed part corresponds to $Q_{\text {HII }} \sim 1$. Lower panel: evolution of the integrated optical depth for the different studies plotted in the upper panel.

the regime where the ionisation fraction is set to unity, which is when ionisation is complete. Finally, in Fig. 1 (lower panel) we plot the Thomson optical depth derived from the ionising background due to star-forming high-redshift galaxies as a function of redshift (dashed lines). We also display the optical depth from the best-fit CMB models (solid lines), together with the one-sigma measurement from PCP15 (shaded areas). As noted in BOU15 and ROB15, star-forming high-redshift galaxies provide enough ionising background to obtain a Thomson optical depth of about 0.06, compatible (on the lower side) with Planck standard $\Lambda \mathrm{CDM}$ constraints $(\tau \sim 0.066 \pm 0.013)$. Here again, the high- $\tau$ value (blue) is definitively at odds with other astrophysical constraints.

\section{New parameterization of the reionisation history}

Current astrophysical constraints seem to favour low values of the optical depth, regardless of the probe, with a sharp reionisation transition between redshifts $\sim 7$ and $\sim 6$. Reionisation histories derived from the abundance of star-forming galaxies suggest an asymmetric behaviour of $Q_{\mathrm{HII}}$ with redshift. On the numerical side, recent simulations reproducing large volumes of the Universe allow for multiple populations of ionising sources, including early Population III stars and self-regulated UV emitting sources (e.g. Ahn et al. 2012; Park et al. 2013). These simulations show that in the most general cases, the reionisation history described in terms of $x_{\mathrm{e}}(z)$ shows a $z$-asymmetric behaviour (see e.g. Fig. 3 in Park et al. 2013).

So far, CMB analyses in turn have assumed a redshiftsymmetric instantaneous reionisation history (implemented e.g. in the publicly available code CAMB, Lewis et al. 2000). This widely used reionisation parameterization is a tanh-based expression $Q_{\mathrm{HII}}(z) \propto\left(1+\tanh \left(\frac{(1+z)^{3}-\left(1+z_{\mathrm{re}}\right)^{3}}{d z}\right)\right) / 2$. The two key parameters, $z_{\text {re }}$ and $d z$, measure the redshift at which the $x_{\mathrm{e}}$ reaches half its maximum (typically 1.08, not including the second reionisation of helium that occurs at $z \sim 3.5$ ) and the duration of reionisation, respectively. In this parameterisation $z_{\mathrm{re}}$ is related one-to-one to $\tau$ for a fixed short duration. As shown in the upper panel of Fig. 1, the shape of the tanh-based expression does not provide a good fit to the data. The middle panel of Fig. 1 shows that the $\rho_{\mathrm{SFR}}$ evolution derived from CMB using a than-based parameterization does not agree with the data, or barely agrees on a rather narrow range of redshifts.

The current astrophysical constraints on reionisation suggest that tanh-based histories have difficulty reproducing the data even in the low- $\tau$ regime. We propose a new parameterisation that allows for a first period where reionisation can be slow and progressive, which is attributable to the softer ionising photons produced by the first stars and primordial dwarf galaxies, and a second period that can be faster leading, by $z \sim 6$, to the completion of hydrogen and first helium reionisation by quasars, for example, with harder ionising photons. This parameterisation is motivated by the behaviour of $Q_{\mathrm{HII}}$ as deduced from simulations and observations. At the end of reionisation, Fan et al. (2006) show that the Gunn-Peterson optical depth evolves as $\tau_{\mathrm{GP}} \propto(1+z)^{\sim 4.3}$. The neutral hydrogen fraction in turn evolves as $\tau_{\mathrm{GP}} /(1+z)^{3 / 2}$, so we consider $1-Q_{\mathrm{HII}} \propto(1+z)^{3}$ at low redshifts below 6. Fan et al. (2006) also note that at redshifts 6 and above, $\tau_{\mathrm{GP}}$ grows much more quickly, possibly $\propto(1+z)^{>11}$. Such an accelerated phase is commonly observed in numerical simulations (Park et al. 2013; Fernandez et al. 2014). We assume that this phase is in fact the end of an exponential phase.

The parameterisation we propose here thus follows these two asymptotic behaviours: a polynomial of order 3 below some pivot redshift $\left(z_{\mathrm{p}}\right)$ and an exponential one above:

$\begin{array}{ll}\text { if } z<z_{p} & 1-Q_{\mathrm{HII}}(z) \propto(1+z)^{3} \\ \text { if } z \geq z_{p} & Q_{\mathrm{HII}}(z) \quad \propto \exp (-\lambda(1+z)) .\end{array}$

The parameterisation is described by three free parameters: the pivot redshift $z_{\mathrm{p}}$, the $Q_{\mathrm{HII}}$-value at the pivot, and the evolution rate in the exponential. Proportionality coefficients can be derived from these three parameters. Depending on the redshift and the $Q_{\mathrm{HII}}$-value at the pivot, the expression is able to reproduce a large family of reionisation histories.

A forthcoming paper (Ilić et al., in prep.) describes the parameterization and its effects on different observations in more detail, in particular the CMB. We show in the upper panel 

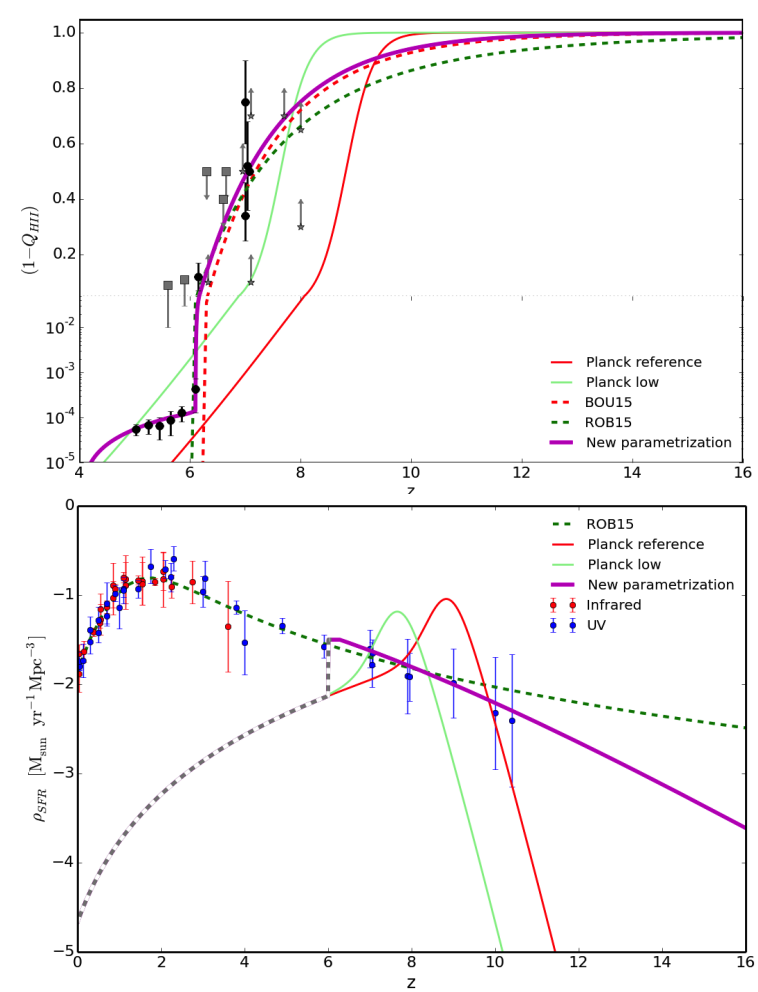

Fig. 2. Upper panel: reionisation histories compared to low-redshift probes. Same as Fig. 1 with the magenta curve displaying the bestfit from the parameterization. Lower panel: same as Fig. 1 with the magenta curve showing the associated derived $\rho_{\mathrm{SFR}}$.

of Fig. 2 the same data points and models as in Fig. 1. We overplot our parameterisation of the reionisation history fitted to the measured values, i.e. excluding lower and upper limits. The corresponding parameter values are: $z_{\mathrm{p}}=6.1, Q_{\mathrm{HII}}\left(z_{\mathrm{p}}\right)=$ $0.99986, \lambda=0.73$. Figure 2 (upper panel) shows that the new parameterisation performs as well as or slightly better than constraints from ROB15 and BOU15 (dashed). It outperforms the tanh-based reionisation expressions used in CMB analyses (solid lines) while having the same value of $\tau$ as the Planck low model and thus quasi-identical EE angular power spectra. However, the redshift of reionisation as defined as $Q_{\mathrm{HII}}\left(z_{\text {reio }}\right)=0.5$ is different in the two approaches: $z_{\text {reio }}=7.05$ for our parameterisation and $z_{\text {reio }}=7.65$ for the tanh expression. Furthermore, the parameterisation reproduces the results from BOU15 in an economical way, with only three free parameters. Using this best fit, we derived the SFR density $\rho_{\text {SFR }}$ from the parameterisation and compared it to the results and compilation from ROB15 and to equivalent CMB-derived values using the tanh expression (Fig. 2 lower panel). In contrast to the latter, the new parameterisation agrees perfectly with the data points and with the ROB 15 results in the redshift range probed by star-forming high-redshift galaxies: it reproduces a slow beginning of reionisation that accelerates to reach an ionisation fraction of about 1 , around the pivot point and a slowly varying ionisation state until the present.

\section{Conclusion}

Recent CMB observations favour lower values of the optical depth, in agreement with values derived from ionising background estimates from star-forming galaxies and lowredshift line-of-sight probes. While this value is weakly sensitive to the modelling of the reionisation history in CMB studies, the latter has so far been assumed to be a simple step transition between neutral and fully reionised hydrogen. This naive parameterisation does not render the behaviour of $Q_{\mathrm{HII}}$ that is observed or derived from other probes. We propose here a new parameterisation motivated by simulations and observations of the reionisation fraction. This new parameterisation reproduces a large family of reionisation studies in an economical way for CMB studies and furthermore yields a good fit to the line-ofsight measurements of the ionised fraction and of the SFR densities. Moreover, it provides a more realistic value of the redshift of reionisation. We thus call for implementing this parameterisation in future CMB analyses for reionisation. Systematic studies of our parameterisation, in particular in $\mathrm{CMB}$ studies, will be presented in a forthcoming paper.

Acknowledgements. The authors thank B.E. Robertson for kindly providing us with his compilation of star formation rate densities. S.I. acknowledges support from the OCEVU Labex (ANR-11-LABX-0060) and the A*MIDEX project (ANR-11-IDEX-0001-02) funded by the "Investissements d'Avenir" French government programme managed by the ANR from LABEX OCEVU. The authors thank the referee for useful comments.

\section{References}

Aghanim, N., Desert, F. X., Puget, J. L., \& Gispert, R. 1996, A\&A, 311, 1 Aghanim, N., Majumdar, S., \& Silk, J. 2008, Rep. Progr. Phys., 71, 066902 Ahn, K., Iliev, I. T., Shapiro, P. R., et al. 2012, ApJ, 756, L16

Bouwens, R. J., Illingworth, G. D., Oesch, P. A., et al. 2015, ApJ, submitted [arXiv: 1503.08228] (IBOU15)

Bowman, J. D., \& Rogers, A. E. E. 2010, Nature, 468, 796

Chornock, R., Berger, E., Fox, D. B., et al. 2014, ApJ, submitted [arXiv: 1405.7400]

Ciardi, B., Ferrara, A., \& White, S. D. M. 2003, MNRAS, 344, L7

Dunlop, J. S., Rogers, A. B., McLure, R. J., et al. 2013, MNRAS, 432, 3520

Ellis, R. S., McLure, R. J., Dunlop, J. S., et al. 2013, ApJ, 763, L7

Faisst, A. L., Capak, P., Carollo, C. M., Scarlata, C., \& Scoville, N. 2014, ApJ, 788,87

Fan, X., Strauss, M. A., Becker, R. H., et al. 2006, AJ, 132, 117

Fernandez, E. R., Zaroubi, S., Iliev, I. T., Mellema, G., \& Jelić, V. 2014, MNRAS, 440, 298

Fontanot, F., Cristiani, S., \& Vanzella, E. 2012, MNRAS, 425, 1413

Gnedin, N. Y. 2000, ApJ, 535, 530

Gruzinov, A., \& Hu, W. 1998, ApJ, 508, 435

Gunn, J. E., \& Peterson, B. A. 1965, ApJ, 142, 1633

Hinshaw, G., Larson, D., Komatsu, E., et al. 2013, ApJS, 208, 19

Ishigaki, M., Kawamata, R., Ouchi, M., et al. 2015, ApJ, 799, 12 (ISHI15)

Kuhlen, M., \& Faucher-Giguère, C.-A. 2012, MNRAS, 423, 862

Lewis, A., Challinor, A., \& Lasenby, A. 2000, ApJ, 538, 473

Loeb, A., \& Furlanetto, S. R. 2013, The First Galaxies in the Universe (Princeton, NJ: Princeton University Press)

Madau, P., \& Dickinson, M. 2014, ARA\&A, 52, 415

Madau, P., Haardt, F., \& Rees, M. J. 1999, ApJ, 514, 648

McQuinn, M., Lidz, A., Zaldarriaga, M., et al. 2009, ApJ, 694, 842

Mitra, S., Choudhury, T. R., \& Ferrara, A. 2011, MNRAS, 413, 1569

Mitra, S., Choudhury, T. R., \& Ferrara, A. 2012, MNRAS, 419, 1480

Mitra, S., Choudhury, T. R., \& Ferrara, A. 2015, MNRAS, submitted [arXiv: 1505.05507]

Page, L., Hinshaw, G., Komatsu, E., et al. 2007, ApJS, 170, 335

Pandolfi, S., Ferrara, A., Choudhury, T. R., Melchiorri, A., \& Mitra, S. 2011, Phys. Rev. D, 84, 123522

Park, H., Shapiro, P. R., Komatsu, E., et al. 2013, ApJ, 769, 93

Planck Collaboration XVI. 2014, A\&A, 571, A16

Planck Collaboration XIII. 2015, A\&A, submitted

[arXiv: 1502.01589] (PCP15)

Planck Collaboration Int. XXX. 2015, A\&A, in press, DOI: $10.1051 / 0004-6361 / 201425034$

Pritchard, J. R., Loeb, A., \& Wyithe, J. S. B. 2010, MNRAS, 408, 57

Robertson, B. E., Ellis, R. S., Dunlop, J. S., McLure, R. J., \& Stark, D. P. 2010 Nature, 468, 49

Robertson, B. E., Furlanetto, S. R., Schneider, E., et al. 2013, ApJ, 768, 71

Robertson, B. E., Ellis, R. S., Furlanetto, S. R., \& Dunlop, J. S. 2015, ApJ, 802, L19 (ROB15)

Spergel, D. N., Verde, L., Peiris, H. V., et al. 2003, ApJS, 148, 175

Stark, D. P., Schenker, M. A., Ellis, R., et al. 2013, ApJ, 763, 129

Willott, C. J., Delorme, P., Reylé, C., et al. 2010, AJ, 139, 906 